\title{
eJRIEPS
}

Ejournal de la recherche sur l'intervention en éducation physique et sport

Numéro spécial 3 | 2020

Actes de la 11 ème Biennale de l'ARIS, Lille, 19-21 juin

2018

\section{Systèmes de valeurs et bien-être psychologique au travail des enseignants d'Éducation Physique et Sportive}

Clément Llena, Isabelle Joing et Jacques Mikulovic

\section{(2) OpenEdition}

Journals

Édition électronique

URL : http://journals.openedition.org/ejrieps/4292

ISSN : 2105-0821

Éditeur

ELLIADD

\section{Référence électronique}

Clément Llena, Isabelle Joing et Jacques Mikulovic, « Systèmes de valeurs et bien-être psychologique au travail des enseignants d'Éducation Physique et Sportive », eJRIEPS [En ligne], Numéro spécial 3 । 2020, mis en ligne le 01 janvier 2020, consulté le 18 février 2020. URL : http://

journals.openedition.org/ejrieps/4292

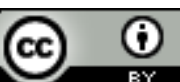

La revue eJRIEPS est mise à disposition selon les termes de la Creative Commons Attribution 4.0 International License. 


\section{Systèmes de valeurs et bien-être psychologique au travail des enseignants d'Éducation Physique et Sportive}

Clément Llena* \&** , Isabelle Joing* \& Jacques Mikulovic ${ }^{* *}$

* Univ. Lille, Univ. Littoral Côte d'Opale, Univ. Artois, EA 7369 - URePSSS - Unité de Recherche Pluridisciplinaire Sport Santé Société, F-59000 Lille, France.

** Univ. Bordeaux, EA 7437 - LACES - Laboratoire Culture Éducation Société, F-33000 Bordeaux, France.

\section{Résumé}

Le bien-être au travail est un nouveau défi social et il n'échappe pas à l'enseignement notamment en Éducation Physique et Sportive (EPS). En effet, des stratégies d'intervention, parfois inconscientes, favorisent le bien-être psychologique au travail des acteurs. L'objet de cette recherche est d'examiner le lien entre le système de valeurs des enseignants d'EPS et leur bien-être psychologique au travail. Leur système de valeurs a été étudié dans différents contextes. 396 enseignants d'EPS de l'Académie de Lille ont participé à cette étude. Les résultats révèlent que les valeurs d'ouverture au changement, de dépassement de soi (dans le sens "pour aller vers les autres ») et d'affirmation de soi sont positivement corrélées au bien-être psychologique au travail. À l'inverse, les valeurs de continuité lui sont négativement corrélées. En fonction des priorités de valeurs de l'enseignant, de ses stratégies d'intervention et mises en œuvre pédagogiques et didactiques (valeurs opérationnalisées en EPS), l'enseignant est donc plus ou moins en bien-être psychologique au travail. Des perspectives s'ouvrent pour la formation initiale et continue des enseignants d'EPS afin de répondre à ces nouveaux enjeux.

Mots-clés : système de valeurs, contexte, responsabilité, Schwartz, enseignement, stratégie d'intervention, EPS

\section{Summary}

Well-being at work is a new challenge for social benefits, and it is no exception to teaching, especially in Physical Education (PE). Indeed, intervention strategies, sometimes unconscious, promote teachers' psychological well-being at work. 


\section{eJRIEPS spécial 3 janvier 2020}

The purpose of this research is to examine the link between values' priorities of PE teachers and their psychological well-being at work. Their values priorities have been studied in different contexts. 396 PE teachers from the "Academy of Lille" participated in this study. The results reveal that the values of openness to change, self-transcendence (that is "going to others") and self-enhancement are positively correlated with psychological well-being at work. Conversely, conservation values are negatively correlated. Depending on teachers' values' priority, their intervention strategies, their pedagogical and their didactic implementations, they are more or less in a condition of psychological well-being at work. Perspectives are opening up for the initial and continuing training of PE teachers to respond to these new major societal challenges.

Key words: value dimensions, context, responsibility, Schwartz, teaching, intervention strategy, physical Education

\section{Introduction}

L'approche managériale prône que « le bonheur est la cause du succès » (Lyubomirsky, King, \& Diener, 2005, p. 803). Pour viser des impacts bénéfiques et élaborer de nouvelles stratégies d'intervention, le bien-être au travail constitue sans doute une piste prometteuse et l'enseignement de l'EPS n'y échappe pas (Schleicher, 2018). Le bien-être et le bonheur des enseignants méritent une attention approfondie, car ils sont susceptibles d'avoir un impact sur le bien-être et l'apprentissage des élèves (Curby, Downer, \& Booren, 2014 ; Rascle \& Bergugnat, 2016). Les relations entre le bien-être au travail des enseignants et le comportement des élèves, voire même leurs performances, sont amplement démontrées (OCDE, 2014 ; Renshaw, Long \& Cook, 2015 ; Rascle \& Bergugnat, 2016 ; Schleicher, 2018). Si les discours actuels de l'institution font du bien-être une mission importante dans la société et l'école, les études sont essentiellement tournées vers les élèves (Ferrière Bacro, Florin, \& Guimard, 2016). Les résultats des travaux montrent notamment, les liens forts entre le bien-être psychologique au travail, le sentiment de compétences et la réussite des élèves (Schleicher, 2018), quelle que soit la nature des relations entre les enseignants et les élèves (Florin \& Guimard, 2017). Par ailleurs, le rapport de l'OCDE (Schleicher, 2018) met en avant que la promotion du bien-être psychologique des enseignants à l'école peut favoriser la santé et le développement socio-émotionnel de tous les élèves. II est même précisé que « pour que l'enseignement soit plus efficace, les enseignants doivent avoir un 


\section{eJRIEPS spécial 3 janvier 2020}

haut niveau de bien-être, de sentiment d'efficacité et de confiance » (Schleicher, 2018, p. 90).

Par ailleurs, un des défis de l'éducation consiste à lutter contre une recrudescence des difficultés professionnelles des acteurs dans l'enseignement (Nunez Moscoso \& Murillo, 2017). Le nombre croissant d'exigences académiques (Bryk, 2017), la prolifération des préconisations descendantes (Rayou, 2017), la gestion «pyramidale et inhumaine » (Debarbieux, 2017, p. 17) amènent une augmentation des conflits et une détérioration des interactions sociales, ces paramètres participent au malaise des enseignants au travail (Barrère, 2017). Les contextes d'intervention (cf. l'École de la confiance) sont en mutation et demandent d'élaborer de nouvelles stratégies pour varier leur enseignement.

Les changements économiques, environnementaux et sociaux du XXle siècle ont poussé les organisations à réfléchir sérieusement à leur propre durabilité et à l'engagement de ses acteurs. Dès lors, le bien-être est une préoccupation majeure des politiques actuelles en matière de santé publique, et plus particulièrement des politiques éducatives à l'école.

Le présent travail rentre dans la dynamique de ces préoccupations en s'intéressant au bienêtre professionnel des professeurs d'EPS, et en l'abordant selon un angle singulier, peu abordé par la littérature, à savoir celui des valeurs. Pour comprendre le bien-être des élèves à l'école, un modèle de « classe prosociale » a été proposé (Eccles \& Roeser, 2011). Les auteurs formalisent que les valeurs constituent un angle possible pour expliquer le bien-être des élèves et leurs compétences psychosociales. Pour expliquer le bien-être professionnel de l'enseignant d'EPS, nous avons choisi de nous situer au niveau 3 du modèle (Eccles \& Roeser, 2011), à savoir celui de l'enseignant lui-même en interrogeant ses propres valeurs. L'objet de ce travail est d'examiner la relation entre le système de valeurs des enseignants d'EPS dans différents contextes (contexte général, contexte professionnel de l'enseignement, contexte de l'EPS) et leur bien-être psychologique au travail afin d'identifier ou non l'existence de valeurs vertueuses permettant d'expliquer le bien-être de l'enseignant d'EPS.

\section{Valeurs et bien-être psychologique au travail}

Pour Reboul (1992, p. 39), « est valeur ce qui vaut la peine». De fait, les valeurs revêtent un caractère polymorphe. Elles sont des principes directeurs qui transcendent les actions et les situations spécifiques. Elles consentent à faire des sélections ou des évaluations d'actions, des personnes, des phénomènes ou des faits (Schwartz \& Bilsky, 1990 ; Schwartz, 2006). Les enseignants peuvent avoir différentes stratégies d'intervention. Ils 


\section{eJRIEPS spécial 3 janvier 2020}

fixent des objectifs, explicites ou implicites, pour leur enseignement (Chang, 2009). En effet, " il n'y a pas d'éducation sans valeurs à transmettre " (Reboul, 2016, p. 1). Dans ce sens, l'enseignement est teinté de valeurs, de motivations éthiques ou intrinsèques (Sahlberg, 2010). Les valeurs s'étudient dans le rapport à une norme, elle-même élaborée à partir d'une « hiérarchie de valeurs » (Reboul, 2016, p. 105). Ce tout structure les valeurs de façon dynamique par rapport à chaque individu sous la forme d'un système de valeurs. Nous voyons la place centrale des valeurs dans la façon d'être, de parler, de ressentir et implicitement, dans la manière d'enseigner.

\subsection{Système de valeurs des individus}

Le modèle théorique de Schwartz $(1992,2006)$ est le plus répandu dans la littérature internationale (Hitlin \& Piliavin, 2004). II a une validité théorique et empirique dans différents domaines (Bardi \& Schwarz, 2013) et notamment pour la mesure des valeurs au travail (Ros, Schwartz, \& Surkiss, 1999). Ce sont « des expressions spécifiques des valeurs générales dans le contexte du travail » (Ros, Schwartz, \& Surkiss, 1999, p. 54). En effet, les valeurs peuvent être mises en actes différemment selon le contexte (De Clercq, Fontaine, \& Anseel, 2008). Ce modèle théorique de Schwartz (Schwartz, 1992 ; Figure 1) développe une structure universelle en dix valeurs de base : l'autonomie, la stimulation, l'hédonisme, la réussite, le pouvoir, la sécurité, la tradition, la conformité, la bienveillance et l'universalisme. Elles s'assemblent en quatre domaines de valeurs (Schwartz, 2006) : l'ouverture au changement, l'affirmation de soi, la continuité et le dépassement de soi. Dans ce circumplex de valeurs, "les individus se distinguent par les priorités qu'ils donnent à ces valeurs » (Schwartz, 2006, p. 929).

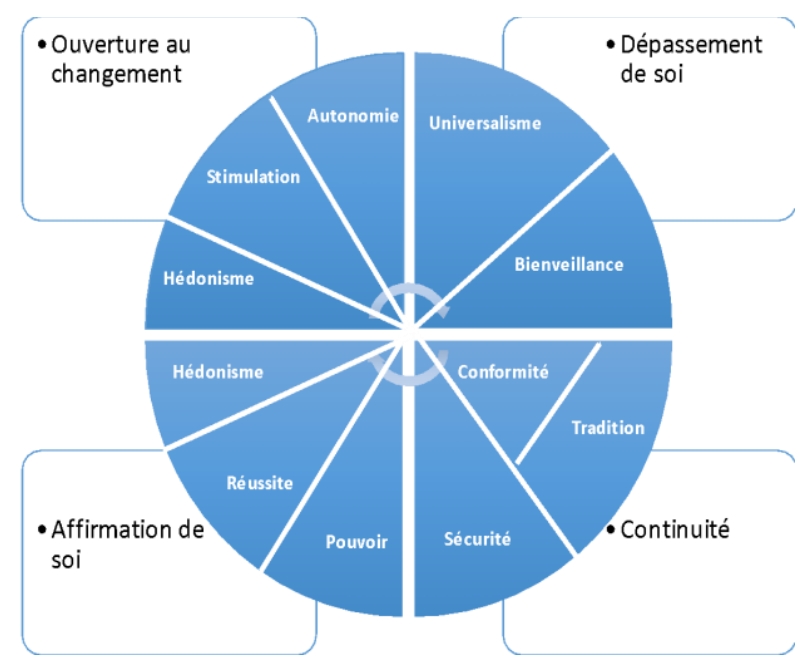

Figure 1: Le modèle des dix valeurs humaines universelles de base agencées en quatre domaines de valeurs (Schwartz, 2006) 
Dans ce diagramme circulaire, les domaines de valeurs s'opposent deux à deux et s'actualisent en fonction de leurs hiérarchies. La représentation spatiale permet de rendre compte de la relation entre les valeurs et leurs domaines respectifs. Par exemple, le domaine de valeurs de l'ouverture au changement est opposé à celui de la continuité.

Par ailleurs, le sexe et l'âge influent sur le système de valeurs. Les femmes et le fait de vieillir donnent une prévalence aux valeurs de continuité (Schwartz, 2006). Une fois adulte, la stabilité du système est renforcée, mais ce dernier reste dynamique selon l'environnement culturel (Schwartz, 1997). Enfin, le système de valeurs peut différer selon le domaine professionnel (Avallone, Farnese, Pepe, \& Vecchione, 2010 ; Consiglio, Cenciotti, Borgogni, Alessandri, \& Schwartz, 2017). Plus spécifiquement, les résultats sur une population d'enseignants révèlent des systèmes de valeurs avec une prévalence sur le domaine de l' « ouverture au changement » par rapport aux autres populations (Schwartz, 1997 ; Schwartz \& Bardi, 2001).

Des travaux basés sur le cadre théorique des « orientations de valeurs » (Jewett, Bain, \& Ennis, 1995) ont permis de montrer le poids des valeurs sur les pratiques d'enseignement de l'EPS (Le Bot, Desbiens \& Pasco, 2016). Par ailleurs, le contexte d'enseignement peut aussi impacter les valeurs (Le Bot, Pasco, \& Desbiens, 2011). Cependant, aucun travail, à notre connaissance n'a cherché à examiner les valeurs en actes des enseignants d'EPS (valeurs opérationnalisées dans le contexte singulier de l'enseignement de l'EPS) avec le cadre théorique de Schwartz.

\subsection{Bien-être au travail}

Le bien-être a été étudié dans un premier temps comme un concept générique applicable dans des domaines de vie spécifique comme le travail (Kashdan, Biwas-Diener, \& King, 2008). La littérature récente montre que le bien-être dans l'activité professionnelle est un concept à part entière (Biétry \& Creusier, 2013). Le bien-être psychologique au travail se situe dans une logique bidimensionnelle entre les deux approches du bien-être: eudémonique et hédonique (Biétry \& Creusier, 2013). L'eudémonisme est plus orienté sur la réalisation de soi, tandis que l'hédonisme est basé sur l'évitement de la souffrance et la recherche du plaisir. Le bien-être psychologique au travail est « une expérience subjective positive où l'on tend à exprimer le meilleur de soi et qui se construit à travers soi, à travers ses relations sociales au travail et dans les interactions avec son organisation » (DagenaisDesmarais \& Privé, 2010, p. 70). Il comprend cinq domaines (Dagenais-Desmarais \& 


\section{eJRIEPS spécial 3 janvier 2020}

Savoie, 2012) : “l'adéquation interpersonnelle au travail ", "l'épanouissement au travail », " le sentiment de compétence au travail », «la volonté d'engagement au travail » et «la reconnaissance perçue au travail ». Le bien-être psychologique au travail de l'enseignant est donc perçu comme l'expression d'une approche globale et systémique (alliant à la fois, la réalisation de soi et la recherche de plaisir) dans son environnement professionnel (Dagenais-Desmarais \& Savoie, 2012).

Des études permettent de faire le constat d'une diminution du bien-être des enseignants en France. En effet, 30\% songent à changer de métier (Guibert, 2017), ils se déclarent isolés (Jégo \& Guillo, 2016), les chiffres montrent un décrochage du métier d'enseignant (Karsenti, Collin \& Dumouchel, 2013). Les professeurs d'EPS ne sont pas préservés par ces difficultés professionnelles (Coutarel et al., 2015). Cependant, peu d'études ont cherché à comprendre ce bien-être en identifiant les facteurs explicatifs du côté de l'individu (système général de valeurs) et de ses pratiques professionnelles (valeurs en actes). Seuls les travaux sur le couplage structurel acteur-environnement (Eccles \& Roeser, 2009) et les congruences entre l'école et l'enseignant permettent d'interroger le bien-être et les valeurs (Skaalvik \& Skaalvik, 2011).

\subsection{Bien-être et valeurs}

Les valeurs sont utilisées comme une boussole pour comprendre le mal-être (Schoëndorff, Grand, \& Bolduc, 2011). Par ailleurs, des recherches (Sagiv \& Schwartz, 2000 ; Sagiv, Roccas, \& Hazan, 2004) montrent des corrélations entre des valeurs (valeurs de réussite, d'autonomie et de stimulation) et le bien-être subjectif. Une recherche plus récente (Sortheix \& Schwartz, 2017) réalisée à partir de données européennes sur une population générale montre que les valeurs d'ouverture au changement sont corrélées positivement avec le bien-être subjectif des individus. À l'inverse, les valeurs de continuité sont négativement corrélées à leur bien-être professionnel. Cependant, il n'y a pas eu de recherches à notre connaissance, sur le lien entre certains domaines de valeurs et le bienêtre au travail dans l'enseignement et encore moins pour des valeurs opérationnalisées dans le contexte professionnel.

L'objet de cette recherche est d'étudier les relations entre les systèmes de valeurs dans différents contextes (système général de valeurs (SVG), système de valeurs au travail (SVens), système de valeurs en actions en EPS (SVeps)) et le bien-être psychologique au travail des professeurs d'EPS. L'identification de domaines de valeurs bénéfiques est une nouvelle focale pour repenser l'acte d'intervention et innover dans les stratégies déployées 


\section{eJRIEPS spécial 3 janvier 2020}

par les acteurs. En fonction des valeurs que l'on défend dans son contexte général, dans le contexte professionnel de l'enseignement ou celui de l'EPS, on serait plus ou moins en bienêtre psychologique au travail. La littérature existante nous permet d'émettre l'hypothèse suivante : la prévalence des valeurs d'ouverture au changement et de dépassement de soi dans ses systèmes de valeurs impacteraient positivement le bien-être psychologique au travail des enseignants d'EPS. À l'inverse, le système de valeurs de la continuité l'impacterait négativement.

\section{Méthodologie}

\subsection{Participants}

396 enseignants d'EPS (196 femmes et 200 hommes) de l'Académie de Lille ont participé à l'étude en avril 2018. L'échantillon représente 19,5\% de la population cible (composée de 2037 professeurs d'EPS provenant des 678 établissements d'enseignement secondaire publics et privés de l'Académie de Lille). Les variables suivantes présentent une bonne variabilité écologique : statut de l'enseignant, ancienneté (l'échantillon présente néanmoins une sur-représentation des fonctionnaires stagiaires), type d'établissement (REP/ sans classification, public/privé). Seules les variables sexe et fonctionnaires stagiaires ne sont pas homothétiques de la population cible. Les femmes $(49,74 \%)$ et les fonctionnaires stagiaires $(6,95 \%)$ sont sur-représentés dans l'échantillon par rapport la population cible $(39,11 \%$ de femmes et $2,26 \%$ de fonctionnaires stagiaires). Toutes les variables ont été contrôlées avec un test de conformité du Khi-deux ( $p>0,05)$.

\subsection{Matériels et procédures}

Un questionnaire unique a été conçu afin de mesurer le SVG (partie 1), le SVGens (partie 2), le SVGeps (partie 3) et le bien-être psychologique au travail (partie 4). La durée de passation était d'environ 12 minutes. Des questions préliminaires permettaient également de recueillir les variables sociologiques.

\subsubsection{Portrait général de valeurs : le PVQ-21 (Schwartz, 2003 - partie 1)}

Les valeurs générales de l'individu ont été interrogées grâce à l'outil validé « de valeurs par portraits » (Schwartz et al., 2001 ; Schwartz, 2003). Cet outil est le plus répandu dans la recherche internationale sur les valeurs (Hitlin \& Piliavin, 2004). Les études scientifiques sur les valeurs utilisent principalement cet outil de mesure (e.g., Cieciuch, Davidov, Algesheimer, \& Schmidt, 2016). II est constitué de 21 items. 


\section{eJRIEPS spécial 3 janvier 2020}

Les assertions sont des affirmations descriptives d'un individu. II faut se comparer à cette personne et répondre dans quelle mesure elle nous ressemble. La phrase introductive du questionnaire est « Je vais maintenant rapidement vous décrire quelques personnes. Écoutez s'il vous plaît chaque description et dites-moi dans quelle mesure cette personne vous ressemble ou pas ». Cette méthodologie permet de limiter le biais de désirabilité sociale (Schwartz et al., 2001). Les réponses se font avec une échelle de type Lickert en six points (de 1 : «pas du tout comme moi » à 6 : « tout à fait comme moi »).

L'outil de mesure est différencié selon le sexe afin que le répondant puisse s'identifier à cette comparaison. L'assertion 1 pour la valeur de l' « hédonisme » qui se caractérise par le domaine de l'ouverture au changement est par exemple : “Avoir du bon temps est important pour lui. Il aime bien se faire plaisir ». Pour la valeur « sécurité » qui se rattache au domaine de la continuité, l'item 1 est «Vivre dans un environnement sécurisant est important pour lui. Il évite tout ce qui peut mettre en danger sa sécurité ».

Le score moyen de chaque individu correspond à la moyenne des scores de réponses. La différence entre le résultat brut de chaque valeur ou domaine de valeurs et ce score moyen permet de calculer un score centré. Ce dernier sera positif ou négatif selon l'importance de chaque valeur dans le SVG. Pour donner un exemple, si le score du domaine de valeurs “ continuité » est de 5,0 et que la moyenne des scores bruts pour l'individu est de 5,5. La différence est donc de - 0,5 pour ce domaine de valeurs. Ce score négatif montre une moinsvalue de ce domaine de valeurs pour l'individu. Chaque score de valeurs dépend avant tout de l'importance accordée à chacune des valeurs en comparaison par rapport aux autres. Les valeurs se hiérarchisent entre elles de façon dynamique dans le SGV.

2.2.2. Valeurs opérationnalisées dans l'enseignement en général : le PVQ-Pro (partie 2)

Ces valeurs ont été déclinées en actions professionnelles pour construire un nouvel instrument de mesure permettant de repérer les valeurs « en actions » des enseignants d'EPS dans leur contexte professionnel d'enseignement (PVQ-Pro). Les items du PVQ-21 (Schwartz, 2003) ont été examinés pour construire des situations de mises en œuvre précises sous la forme de «courts portraits» de valeurs opérationnalisées dans l'enseignement en général. Les répondants peuvent alors se comparer à ces actions et mesurer les systèmes de valeurs dans le contexte professionnel d'enseignement. De fait, les items du PVQ-21 (Schwartz, 2003) ont été traduits en indicateurs de valeurs, en indices, en items concrets puis enfin en assertions opérationnalisées dans l'enseignement en 


\section{eJRIEPS spécial 3 janvier 2020}

général. Par exemple, pour le domaine de l'«ouverture au changement» et plus spécifiquement la valeur de l' " hédonisme », l'assertion 1 est: "Dès que ses cours se terminent, il quitte l'établissement pour gérer ses impératifs personnels ». Pour la valeur « sécurité », l'item 1 est : «L'environnement de l'établissement (le quartier) est un critère déterminant lors de sa demande de mutation intra-académique ».

En continuité avec le PVQ, chaque item incite à se comparer à l'action d'un individu. Le répondant se positionne de façon à savoir dans quelle mesure il est en accord avec les assertions proposées. Chacune correspond à une valeur de base opérationnalisée en actes dans le contexte d'enseignement. L'échelle de réponse propose 6 possibilités (de 1: «Pas du tout comme moi » à 6 : «Tout à fait comme moi »). Les qualités psychométriques de cet outil ont été testées. L'alpha de Cronbach est de 0,735 (supérieurs au score minimal de 0,70, établi par Schwartz $(2003,2006)$ ou 0,65 dans la littérature en statistique (Carricano, Poujol, \& Bertrandias, 2010). Le modèle à quatre facteurs de l'échelle a montré une bonne adéquation globale avec l'ensemble de données : $\chi^{2}(108)=174.17 ; p=0.012 ; \chi^{2} / \mathrm{df}=$ $1.08 ; \mathrm{CFI}=0.943 ; \mathrm{RMSEA}=0.057 ; \mathrm{SRMR}=0.079$. Les résultats indiquent un bon ajustement aux données (Hu \& Bentler, 1999 ; Byrne, 2001 ; Hooper, Coughlan, \& Mullen, 2008). Cet outil de mesure respecte les critères de validité, de fiabilité, la cohérence structurelle et de sensibilité.

\subsubsection{Valeurs opérationnalisées en EPS : le PVQ-EPS (partie 3)}

De la même façon, un instrument de mesure a été développé pour repérer les valeurs « en actions » des enseignants d'EPS dans le contexte spécifique de l'EPS (PVQ-EPS). Dès lors, les assertions du PVQ-21 ont été opérationnalisées dans les pratiques liées à l'EPS. Par exemple, pour le domaine de l' « ouverture au changement » et plus spécifiquement la valeur de « hédonisme », l'assertion 1 est : «Pendant ses cours, il pratique avec ses élèves, cela lui fait plaisir ». Pour la valeur de « sécurité » (domaine de valeurs de la « continuité »), l'item 1 est : « I/ hésite à faire des sorties d'APPN par peur d'un incident ».

De la même façon, ces items permettent de se comparer à quelqu'un et d'interroger dans quelle mesure la personne est d'accord avec les affirmations de priorités de valeurs professionnelles énoncées pour cette personne. L'échelle de réponse propose 6 possibilités (de 1 : «Pas du tout d'accord » à 6 : «Tout à fait d'accord »).

Les qualités psychométriques de cet outil ont été testées. L'alpha de Cronbach est de 0,739 (seuil acceptable ; op.cit.). Le modèle à quatre facteurs de l'échelle a montré une très bonne adéquation avec l'ensemble de données : $\chi^{2}(146)=191.89 ; p=0.006 ; \chi^{2} / \mathrm{df}=1.31 ; \mathrm{CFI}$ 
eJRIEPS spécial 3 janvier 2020

$=0.95 ; \mathrm{RMSEA}=0.04 ; \mathrm{SRMR}=0.06$. Les résultats indiquent un ajustement aux données satisfaisant (op.cit.). II respecte les critères de validité, de fiabilité, la cohérence structurelle et de sensibilité.

2.2.4. Bien-être psychologique au travail : I'IBEPT (Dagenais-Desmarais \& Savoie, 2012 - partie 4)

L'outil de mesure utilisé pour mesurer le bien-être psychologique au travail est l'« Index de Bien-être Psychologique au Travail » (IBEPT). Il a été validé par Dagenais-Desmarais et Savoie (2012). II possède une bonne assise théorique dans la littérature scientifique (Loup, 2016). L'outil permet de mesurer cinq dimensions ("adéquation interpersonnelle au travail », "épanouissement dans le travail », "sentiment de compétence au travail », « reconnaissance perçue au travail », et « volonté d'engagement au travail ») en 25 items. Même si ce modèle en cinq dimensions témoigne d'une prédominance pour le caractère eudémonique du bien-être au travail, certains items interrogent la dimension hédonique avec des états affectifs (e.g., " J'aime mon travail »). L'approche est combinée et mesure à la fois le bien-être hédonique et le bien-être eudémonique (Dagenais-Desmarais \& Savoie, 2012). Chaque assertion décrit comment les individus peuvent se sentir au travail. L'échelle de réponse est de type Lickert en 6 points (de 1 : «En désaccord "; à 6 : "Tout à fait en accord "). II demande d'évaluer son expérience au travail au cours des quatre dernières semaines. L'assertion 1 pour le domaine de «volonté d'engagement au travail » est : « J'ai envie de prendre des initiatives dans mon travail ». Le score de bien-être psychologique au travail est calculé par dimension et/ou avec un score total. lls représentent la moyenne des items référents de chacun des indicateurs. Plus le résultat se rapproche de 6 , plus l'enseignant satisfait cet indicateur.

\subsection{Procédure}

Un recrutement large des enseignants d'EPS de l'Académie de Lille a été visé afin d'avoir différents profils de répondants. Le choix d'une passation en ligne a permis de prendre contact avec de multiples réseaux et notamment l'inspection régionale en EPS et le rectorat de l'Académie de Lille. En effet, une diffusion par voie hiérarchique a été préférée pour toucher tous les enseignants d'EPS de l'Académie de Lille. Ainsi, les inspecteurs pédagogiques régionaux ont soutenu cette recherche puis l'ont diffusée sur le site académique en EPS de l'Académie de Lille. Dès lors, tous les chefs d'établissement de l'Académie de Lille ont été contactés pour transférer l'enquête aux professeurs d'EPS de 


\section{eJRIEPS spécial 3 janvier 2020}

leurs établissements. Un courriel type a été construit dans lequel il existait un lien hypertexte actif qui dirigeait le répondant automatiquement vers le questionnaire de la plateforme SphinxOnline et le site EPS de l'Académie de Lille. Le courriel type d'envoi a été rédigé de manière concise et ciblée en précisant qu'il s'agissant d'une étude sur le bien-être au travail des enseignants d'EPS. Le titre de l'enquête dans l'Académie de Lille a été formulé ainsi : "Quel bien-être au travail pour les enseignants d'EPS de l'académie de Lille ? ». Un nombre important d'enseignants en EPS ont ainsi été contactés par cette enquête de recherche (2 relances ont été mises en place à 2 semaines d'intervalle) menée en mars/avril 2018.

Cependant, tous les chefs d'établissements n'ont pas confirmé le transfert aux enseignants d'EPS de leur établissement. Afin de limiter le biais de sélection de l'échantillonnage et afin de le rendre au plus proche de l'échantillon probabiliste, une diffusion au travers d'autres canaux comme l'Association des Enseignants d'Éducation Physique et Sportive (AEEPS), le Syndicat National en Éducation Physique (SNEP), le Syndicat National de l'Enseignement Catholique, le Syndicat Général de l'Education Nationale de la CFDT Nord / Pas de Calais (SGEN CFDT), I'Union Nationale du Sport Scolaire, I'Union Générale Sportive de l'Enseignement Libre (UGSEL) et la formation à l'agrégation interne en EPS de l'Académie de Lille a été envisagée. Ces différents réseaux ont eux aussi partagé l'enquête au travers de leur liste de diffusion (et deux relances ont été effectuées à deux semaines d'intervalle). Enfin, la section académique du SNEP-Lille a laissé un lien en accès libre sur leur page d'accueil pendant la durée de l'étude.

Les informations renseignées ont fait l'objet d'un traitement informatisé déclaré à la correspondante Informatique et Libertés de l'Université de Bordeaux avec le numéro CNIL 2140484. Parallèlement le Comité d'Éthique pour la Recherche en STAPS (CERSTAPS) a été contacté. Un avis favorable a été donné par le comité sous le numéro 2018-27-03-24.

\subsection{Traitements et analyses}

Les préconisations des auteurs ont été suivies pour utiliser les données des questionnaires. Bilsky, Janik, et Schwartz (2011) présentent une " correction d'échelle " pour calculer un score centré en fonction de l'importance accordée à la valeur par rapport à la moyenne des scores de réponses. Dagenais-Desmarais et Savoie (2012) proposent d'utiliser le score global pour l'IPBET. La normalité a été vérifiée avec un histogramme, une droite de Henry et un test de Kolmogorov-Smirnov pour chacune des variables.

Les données ont été traitées avec le logiciel $\mathrm{R}$ studio version 1.1.463 pour les analyses factorielles en composantes principales, l'analyse par clusters et les régressions multiples 


\section{eJRIEPS spécial 3 janvier 2020}

(analyse avec le package «random forest» et le package «boruta»). Le seuil de significativité a été fixé à $p=0.05$.

\section{Résultats}

\subsection{Système de valeurs des enseignants d'EPS : aucune différence significative} selon les contextes (SVG, SVens, SVeps)

Les résultats confirment la structure des relations entre les domaines de valeurs (opposition des domaines deux à deux) du modèle théorique de Schwartz (1992; 2006). Les résultats (Figure 2) révèlent que leurs priorités de valeurs sont les suivantes (dans l'ordre décroissant d'importance) : le domaine de valeur du «dépassement de soi », puis l' "ouverture au changement », la « continuité » et enfin l' «affirmation de soi ». Le tableau I montre de légères disparités entre les valeurs des enseignants d'EPS dans le contexte général (valorisation de l'affirmation de soi et « dévalorisation » de l'ouverture au changement) et celles mises en actes dans son contexte professionnel (valorisation de l'ouverture au changement et « dévalorisation » de l'affirmation de soi).

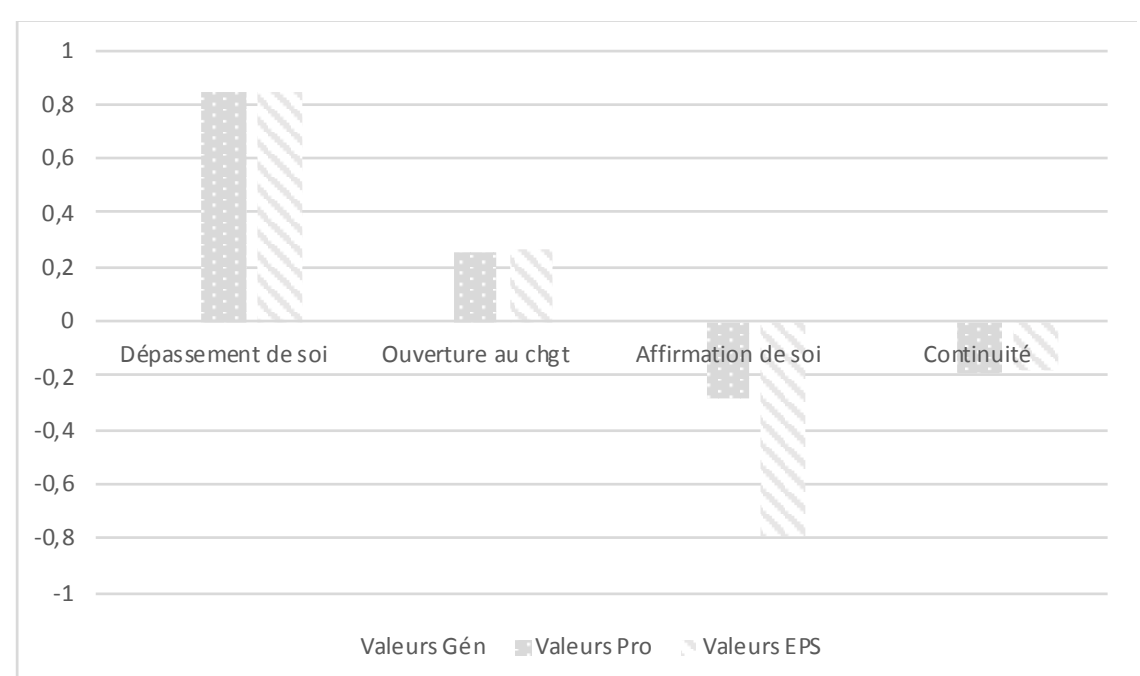

Figure 2 : Histogramme groupé des systèmes de valeurs centrés ((SVG - première colonne en gris), SVens (deuxième colonne en gris pointillé blanc) et SVeps (troisième colonne en blanc hachuré gris)) des enseignants d'EPS de l'Académie de Lille

Par ailleurs, il n'y a pas de différence significative $(p>0.05)$ concernant les systèmes de valeurs dans les différents contextes (SVG, SVGens, SVGeps) pour les professeurs d'EPS de l'Académie de Lille. 


\section{eJRIEPS spécial 3 janvier 2020}

3.2. L'ouverture au changement et le bien-être psychologique au travail des enseignants d'EPS : une association vertueuse

Une analyse en composantes principales associée à une analyse par clusters a été réalisée avec l'ensemble des données quantitatives. Elles permettent d'identifier trois profils d'enseignants d'EPS (Figure 4). Les résultats montrent que le domaine de valeurs de l'ouverture au changement et le bien-être psychologique au travail sont projetés sur le même axe. La Figure 3 représente la distribution des variables sur les axes.

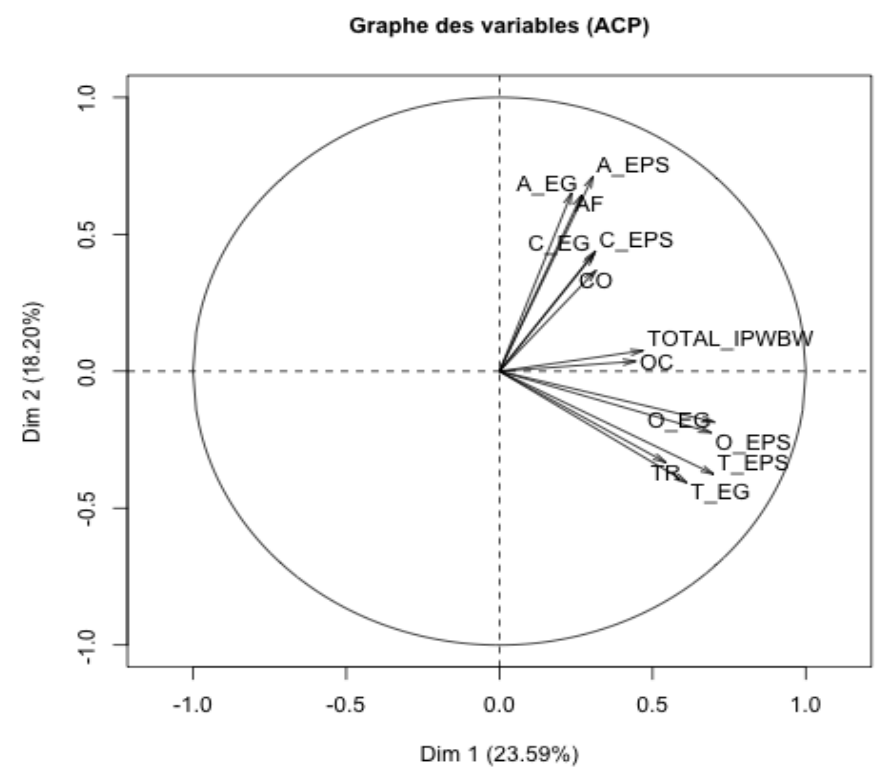

Figure 3 : Organisation des variables sur le premier plan factoriel

Les résultats issus de l'ACP montrent que les dimensions 1 et 2 expliquent $41,79 \%$ de la variance totale (respectivement $23,59 \%$ pour la dimension 1 et $18,20 \%$ pour la dimension 2). La représentation graphique de ces deux dimensions (Figure 3) suggère que le bien-être psychologique au travail et les valeurs d'ouverture au changement dans le SGV sont bien projetés sur l'axe 1, tandis que les autres domaines de valeurs, quel que soit le contexte, sont projetés sur les deux axes. II est à noter que les domaines de l'affirmation de soi et de la continuité sont projetés dans la partie supérieure de l'axe 2 à l'inverse du domaine de l'affirmation de soi.

Des clusters permettent de voir si une typologie d'enseignants semble se distinguer au niveau du bien-être psychologique au travail. Dans ce cas, certaines variables seraient plus caractéristiques des professeurs d'EPS en situation de bien-être. 


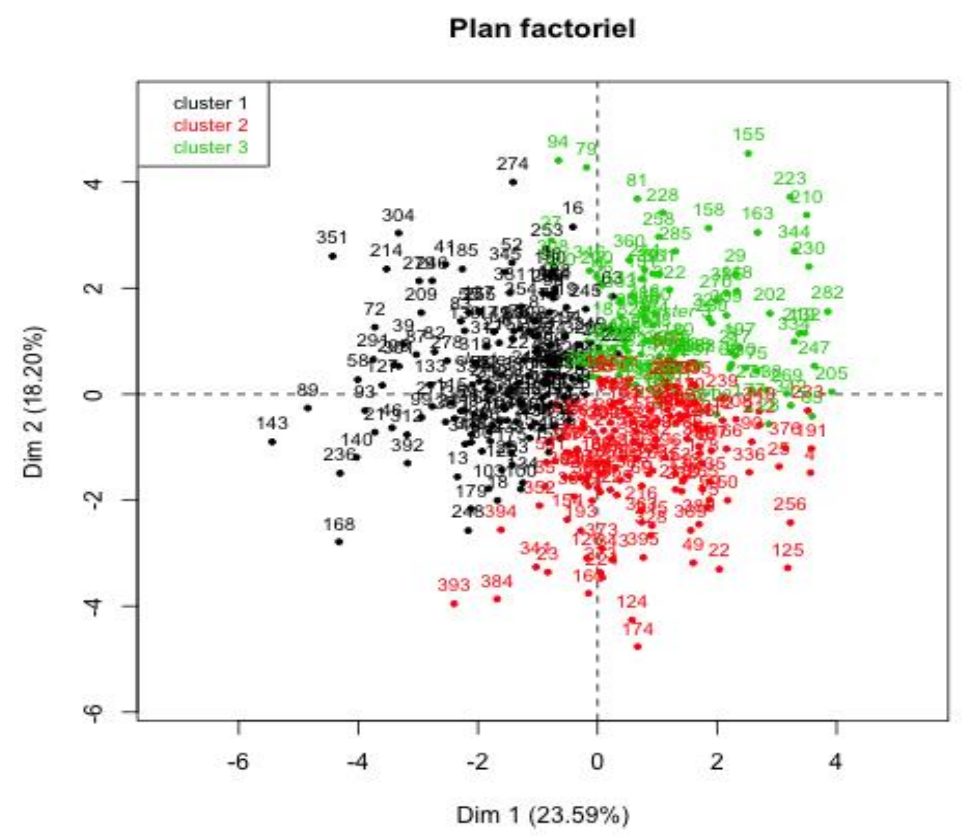

Figure 4 : Répartition de 3 clusters dans un plan factoriel à deux dimensions

L'analyse par clusters met en évidence trois groupes de sujets que l'on peut distinguer essentiellement sur le premier axe de l'ACP (Figure 4). Le premier cluster que l'on nommera les "professeurs d'EPS traditionnels " (à gauche de l'axe), se caractérise par un faible bien-être psychologique au travail, une dominance du domaine de valeurs "continuité " dans le contexte général par rapport au reste du groupe et moins d'importance à ceux d' " ouverture au changement » et du « dépassement de soi ».

Les clusters 2 et 3 (à droite de l'axe) rassemblent des professeurs d'EPS davantage en bien-être psychologique au travail, avec une dominance du domaine de valeurs d' " ouverture au changement " (et donc une moins-value de la continuité). Le domaine du " dépassement de soi » est plus important dans le cluster 2 (comparativement au cluster 3). L' " affirmation de soi » est plus importante dans le cluster 3 (comparativement au cluster 2). Les sujets du cluster 2 seront nommés les “altruistes " et ceux du cluster 3, les "glorieux ». La synthèse des variables caractérisant les clusters est présentée dans le 


\section{Tableau I.}

Tableau I : Présentation des clusters selon les variables significativement différentes des moyennes de l'ensemble de l'échantillon.

\begin{tabular}{|c|c|c|c|c|}
\hline & $\begin{array}{c}\text { Cluster } 1 \\
\text { Les traditionnels }\end{array}$ & $\begin{array}{c}\text { Cluster } 2 \\
\text { Les altruistes }\end{array}$ & $\begin{array}{l}\text { Cluster } 3 \\
\text { Les glorieux }\end{array}$ & $\begin{array}{l}\text { Moyenne } \\
\text { générale }\end{array}$ \\
\hline \multicolumn{5}{|c|}{ Bien-être psychologique au travail } \\
\hline IBEPT & $3,23 \pm 0,68$ & $3,93 \pm 0,63$ & $3,94 \pm 0,65$ & $3,70 \pm 0,65$ \\
\hline \multicolumn{5}{|c|}{ Système général de valeurs (SVG) } \\
\hline Ouverture au changement & $3,81 \pm 0,53$ & $4,32 \pm 0,43$ & $4,31 \pm 0,49$ & $4,11 \pm 0,48$ \\
\hline Affirmation de soi & NS & $3,00 \pm 0,52$ & $3,65 \pm 0,48$ & $3,26 \pm 0,50$ \\
\hline Continuité & $4,41 \pm 0,40$ & $3,81 \pm 0,39$ & $3,81 \pm 0,38$ & $3,97 \pm 0,40$ \\
\hline Dépassement de soi & $4,58 \pm 0,42$ & $5,14 \pm 0,36$ & $5,01 \pm 0,37$ & $4,91 \pm 0,47$ \\
\hline \multicolumn{5}{|c|}{$\begin{array}{l}\text { Système de valeurs dans le } \\
\text { contexte du travail (SVens) }\end{array}$} \\
\hline Ouverture au changement & $3,85 \pm 0,55$ & $4,63 \pm 0,50$ & $4,43 \pm 0,53$ & $4,27 \pm 0,53$ \\
\hline Affirmation de soi & NS & $3,16 \pm 0,63$ & $3,78 \pm 0,60$ & $3,41 \pm 0,61$ \\
\hline Continuité & $4,49 \pm 0,41$ & $3,91 \pm 0,48$ & $3,99 \pm 0,45$ & $4,10 \pm 0,47$ \\
\hline Dépassement de soi & $4,59 \pm 0,49$ & $5,27 \pm 0,43$ & $5,10 \pm 0,48$ & $4,96 \pm 0,48$ \\
\hline \multicolumn{5}{|c|}{$\begin{array}{l}\text { Système de valeurs dans les actes } \\
\text { en EPS (SVeps) }\end{array}$} \\
\hline Ouverture au changement & $3,82 \pm 0,56$ & $4,66 \pm 0,51$ & $4,40 \pm 0,54$ & $4,27 \pm 0,53$ \\
\hline Affirmation de soi & NS & $2,96 \pm 0,56$ & $3,57 \pm 0,48$ & $3,19 \pm 0,53$ \\
\hline Continuité & $4,19 \pm 0,42$ & $3,66 \pm 0,40$ & $3,71 \pm 0,42$ & $4,19 \pm 0,41$ \\
\hline Dépassement de soi & $4,49 \pm 0,43$ & $5,18 \pm 0,37$ & $5,00 \pm 0,44$ & $4,86 \pm 0,45$ \\
\hline
\end{tabular}

IBEPT = Indice de Bien-Etre Psychologique au Travail

$N S$ = représente des valeurs non significativement différentes des moyennes générales

Les clusters 2 et 3 se caractérisent par des enseignants avec un bien-être psychologique au travail significativement supérieur à la moyenne générale, alors que le résultat est inversé pour le cluster 1 . On constate que c'est la dialectique entre l'ouverture au changement et la continuité qui impacte davantage le bien-être psychologique au travail que celle entre les domaines de l'affirmation de soi et du dépassement de soi dans les différents contextes (général, de l'enseignement et de EPS). Ces résultats laissent à penser que les priorités de valeurs impactent le bien-être psychologique au travail des enseignants d'EPS. Plus précisément, ils montrent que l'ouverture au changement est une valeur positivement associée au bien-être des enseignants d'EPS. 


\section{eJRIEPS spécial 3 janvier 2020}

3.3. L'ouverture au changement à l'échelle de l'individu (contexte général) et le dépassement de soi à l'échelle des mises en œuvre en EPS: des variables explicatives du bien-être psychologique au travail.

Certaines valeurs semblent être caractéristiques des individus en bien-être au travail. Les résultats d'une analyse par « random forest » montrent que les deux critères les plus «puissants» pour les enseignants d'EPS en bien-être psychologique au travail sont l'ouverture au changement dans le contexte général et le dépassement de soi dans le contexte de I'EPS (Tableau II).

Tableau II : Classement des domaines de valeurs pour expliquer le bien-être psychologique au travail des professeurs d'EPS.

\begin{tabular}{ll}
\hline & $\begin{array}{c}\text { Importance de chaque } \\
\text { variable explicative }\end{array}$ \\
\hline OC & 18,06 \\
T_EPS & 17,77 \\
AF & 16,22 \\
A_EG & 15,65 \\
C_EG & 15,55 \\
A_EPS & 15,14 \\
T_EG & 15,04 \\
C_EPS & 14,17 \\
O_EPS & 13,36 \\
O_EG & 12,36 \\
CO & 11,98 \\
TR & 11,46 \\
\hline OC = domaine de valeurs de l'ouverture au changement. \\
T = domaine de valeurs du dépassement de soi \\
A = domaine de valeurs de l'affirmation de soi \\
C = domaine de valeurs de la continuité \\
EG = contexte de l'enseignement en général \\
EPS = contexte de l'enseignement en EPS
\end{tabular}

3.4. La dialectique du dépassement de soi/affirmation de soi dans le contexte de I'EPS : une association prédictive du bien-être psychologique au travail

Si des valeurs sont caractéristiques des individus en bien-être psychologique au travail, la méthode de l'apprentissage supervisé a été utilisée pour prédire le bien-être psychologique 


\section{eJRIEPS spécial 3 janvier 2020}

au travail des enseignants d'EPS avec une technique de randomisation. Cette méthode d'apprentissage montre que de manière aléatoire (Kursa \& Rudnicki, 2010) les résultats réellement importants et statistiquement valides diffèrent : les variables de dépassement de soi et d'affirmation de soi dans le contexte de l'EPS sont les variables randomisées les plus importantes pour prédire le bien-être psychologique au travail (Tableau III).

Tableau III. Importance des différents indicateurs quantitatifs pour prédire le bien-être psychologique au travail.

\begin{tabular}{lll}
\hline Variable & $\begin{array}{l}\text { Mean } \\
\text { importance }\end{array}$ & Decision \\
\hline OC & 3.77 & Confirmed \\
AF & 5.06 & Confirmed \\
CO & -1.01 & Rejected \\
TR & 2.79 & Confirmed \\
O_EPS & 4.56 & Confirmed \\
A_EPS & 8.53 & Confirmed \\
C_EPS & 0.6 & Rejected \\
T_EPS & 12.24 & Confirmed \\
O_EG & 5.18 & Confirmed \\
A_EG & 2.35 & Confirmed \\
C_EG & 1.84 & Rejected \\
T_EG & 6.13 & Confirmed \\
\hline
\end{tabular}

La Figure 5 et le Tableau III montrent que les domaines de valeurs de l'ouverture au changement, de l'affirmation de soi et du dépassement de soi ont contribué à prédire le bien-être psychologique au travail de manière significative dans tous les contextes étudiés (général, de l'enseignement, de l'EPS). À l'inverse, le domaine de valeurs de la continuité ne permet pas de prédire le bien-être psychologique au travail dans les différents contextes. 


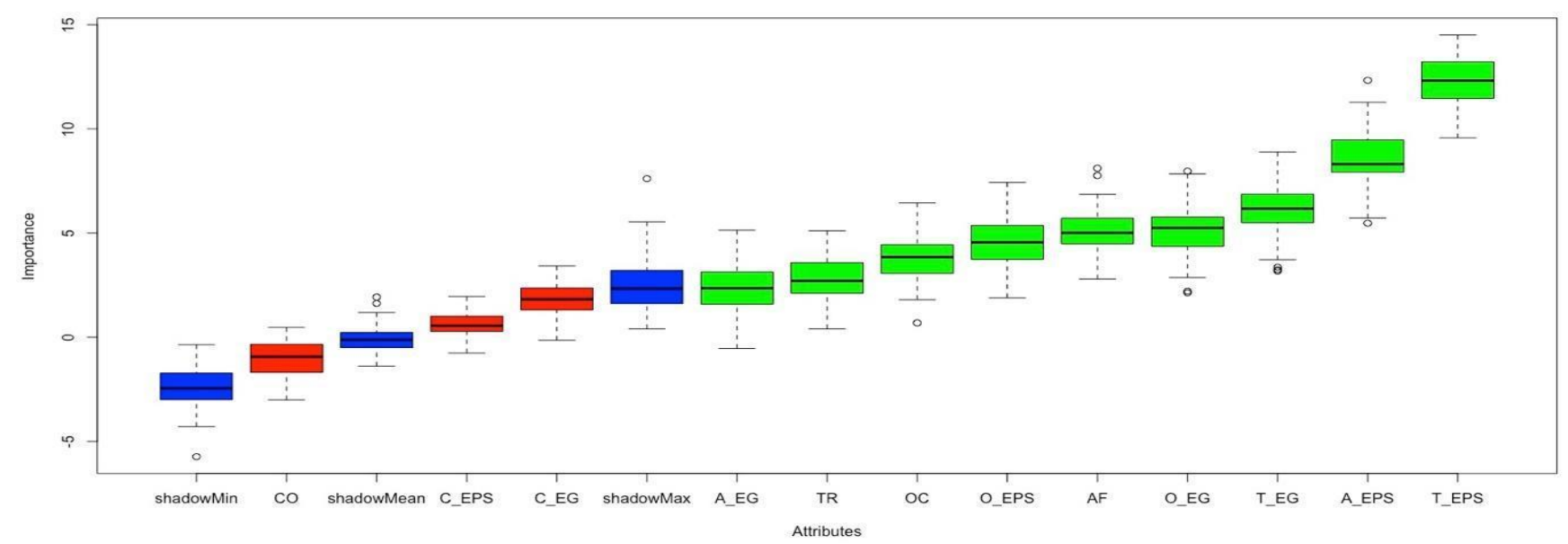

Figure 5: Importance des différents domaines de valeurs pour prédire le bien-être psychologique au travail des enseignants d'EPS (à partir d'une analyse d'arborescence aléatoire)

C'est le dépassement de soi dans le contexte de l'EPS qui contribue le plus au bien-être psychologique au travail, suivi de l'affirmation de soi dans le contexte de l'EPS. La contribution des autres variables de ces mêmes domaines est légèrement supérieure à la variable aléatoire, mais elles sont toujours incluses dans le modèle.

\section{Discussion}

Les résultats montrent qu'il existe des relations entre les systèmes de valeurs dans différents contextes (général, dans l'enseignement, en EPS) des enseignants d'EPS et leur bien-être psychologique au travail. Dès lors, en fonction des systèmes de valeurs de l'enseignant, de ses stratégies d'intervention et de ses mises en œuvre en EPS, l'enseignant est plus ou moins en bien-être psychologique au travail toutes choses égales par ailleurs (sexe, statut de l'enseignant, ancienneté, type d'établissement).

\subsection{Le bien-être psychologique au travail est fonction de ce que l'on fait et des} valeurs que l'on défend

Les résultats ont montré que les valeurs d'ouverture au changement, de dépassement de soi et d'affirmation de soi impactent positivement le bien-être psychologique au travail des enseignants d'EPS. À l'inverse, les valeurs de continuité sont un critère de dépréciation de ce même bien-être. Certaines valeurs sont donc vertueuses et semblent expliquer le bienêtre psychologique au travail des enseignants d'EPS. 


\section{eJRIEPS spécial 3 janvier 2020}

Ces résultats montrent qu'en fonction de son SVG, son SVens et de son SVeps, les enseignants d'EPS sont plus ou moins en bien-être psychologique au travail. Ces éléments sont d'autant plus intéressants par rapport à la littérature récente qui montre qu'il faut investir sur le bien-être psychologique au travail pour viser la performance, la productivité, l'efficience, la qualité, la compétitivité, la culture, le climat de travail et le développement de l'entreprise (Ochoa \& Blanch, 2018).

\subsection{Des valeurs protectrices du bien-être psychologique au travail :} l' « ouverture au changement », le « dépassement de soi » et l' " affirmation de soi » Les analyses par composantes principales et par clusters ont montré que 36 variables sur 39 étaient significativement différentes des valeurs moyennes. Par ailleurs, le domaine de valeurs de l'ouverture au changement et du bien-être psychologique au travail sont projetés sur le même axe et ont permis de distinguer les enseignants d'EPS entre eux. Trois typologies identifiables sont ressorties de cette analyse par clusters : les professeurs d'EPS « traditionnels », caractérisés par un score de bien-être psychologique au travail plus faible que le reste de l'échantillon, les professeurs d'EPS «altruistes » valorisant davantage les valeurs de dépassement de soi et les professeurs d’EPS « glorieux » caractérisés par une prévalence pour les valeurs d'affirmation de soi. Ces deux derniers clusters se différencient avec un score de bien-être psychologique au travail plus important que les « traditionnels ». Ces typologies montrent que les enseignants qui ont un système de valeurs à prévalence pour l'ouverture au changement, le dépassement de soi et l'affirmation de soi sont davantage en bien-être psychologique au travail que les autres à contexte équivalent. Ces résultats sont conformes à la littérature sur les relations entre les valeurs et le bien-être subjectif dans d'autres contextes professionnels (Sagiv \& Schwartz, 2000 ; Sagiv et al. 2004 ; Sortheix \& Schwartz, 2017). Plus spécifiquement, les résultats de Sortheix et Schwartz (2017) ont révélé que l' « ouverture au changement » est positivement corrélée avec le bien-être subjectif. À l'inverse, le domaine de valeurs de la «continuité » lui était négativement corrélé. La présente étude sur les valeurs dans différents contextes complète ces résultats pour favoriser le bien-être psychologique dans l'enseignement de l'EPS.

4.3. Les valeurs des enseignants «altruistes» et «glorieux» sont émancipatrices dans le contexte de l'EPS

Si la prédominance de l'ouverture au changement est une variable reconnue comme prédictive dans la littérature, une analyse par randomisation a montré que les variables de 


\section{eJRIEPS spécial 3 janvier 2020}

dépassement de soi et d'affirmation de soi dans le contexte de l'EPS sont les variables les plus «puissantes » pour expliquer le bien-être psychologique au travail. Dit autrement, les enseignants d'EPS qui mettent en avant dans leurs actes professionnels de l'intérêt pour les autres et s'intéressent à leur bien-être ou qui développent des actions centrées sur leur propre réussite et leurs propres intérêts dans l'enseignement de l'EPS sont des indicateurs favorables pour développer un bien-être psychologique au travail. Ce résultat novateur permettant l'identification de domaines de valeurs bénéfiques est une nouvelle focale pour repenser l'acte d'intervention et dynamiser le processus d'innovation à déployer par les acteurs dans la mesure où il est possible d'agir et de faire évoluer son propre système de valeurs. Cependant, les enseignants en burnout font une utilisation accrue de pratiques d'enseignement orientées vers la performance (Retelsdorf, Butler, Streblow, \& Schiefele, 2010), ce qui a pour conséquence l'établissement de relations sociales négatives (Tolmie et al., 2010) et des progrès limités dans les habiletés conceptuelles des élèves (Fraser, 1998 ; Gillies \& Ashman, 2003). En ce sens, la littérature fait ressortir que les valeurs d'affirmation de soi ne sont pas toujours positives pour les enseignants.

\subsection{La dynamique des valeurs}

S’il y a eu débat dans la littérature (Assael, 1984), les valeurs sont aujourd'hui perçues comme étant à la fois stables et dynamiques. En effet, il fait consensus que les valeurs forment un système et qu'elles sont interdépendantes (Rezsohazy, 2006). Dans la théorie de Schwartz (Schwartz \& Bilsky, 1987, 1990 ; Schwartz 1992), la structure des valeurs de base est stable (cf. la théorie des valeurs de base, Schwartz, 2006), mais des processus influent sur les valeurs à l'intérieur (les priorités de valeurs individuelles évoluent). Fisher et Schwartz (2010) insistent sur la variabilité dans les valeurs des individus. Les valeurs s'ancrent dans un processus interagissant pour expliquer la structure circulaire du système de valeurs (Schwartz, 2006). Plusieurs valeurs peuvent intervenir simultanément dans les actions et les décisions avec des relations d'antagonisme (opposition dans le circumplex de Schwartz) ou de compatibilités (proximité dans le circumplex).

Si d'un côté, des auteurs ont affirmé que « nos actions sont guidées par des valeurs, des normes qui sont loin d'être naturelles, elles sont desconstructions culturelles et symboliques »(Bruner, 2014, p. 38). Un autre pan de la littérature admet que le système de valeurs n'est plus totalement hérité (Galland \& Lemel, 2006). L'agencement des valeurs peut évoluer au gré des expériences, du contexte, de la période, de la culture des individus (Schwartz et al., 2012). Dans une certaine mesure, le système de valeurs est dynamique 


\section{eJRIEPS spécial 3 janvier 2020}

(Schwartz et al., 2012). En psychologie, pour Wilson et DuFrene (2009, p. 66), « elles sont même en constante évolution". Les valeurs sont liées au comportement et aux attitudes. Les travaux sur les orientations de valeurs en EPS (Le Bot et al., 2016) et leurs identifications ont montré l'importance des valeurs sur les contenus enseignés, la manière dont ils sont enseignés. Cette focale semble aussi intéressante pour étudier le bien-être.

\subsection{Implications pour la formation initiale et continue des enseignants}

Les résultats ne peuvent pas être généralisés à tous les enseignants, mais ils souscrivent à une nouvelle réflexion pour repenser l'acte d'intervention en EPS. In fine, ils permettent d'envisager des pistes intéressantes pour la formation initiale et continue des enseignants. Un enseignant d'EPS est en bien-être psychologique au travail, s'il développe sa stimulation et son autonomie, ou des pratiques basées sur l'intérêt pour les autres ou, encore, ses propres intérêts de manière intentionnelle. Au contraire, l'enseignant a un score de bienêtre psychologique au travail moins élevé s'il développe des pratiques d'intervention proches de celles qu'il connaît déjà. Dans un premier temps, il semble essentiel de prendre connaissance et d'incorporer ces éléments dans la formation initiale et continue. Ces préconisations laissent de nombreuses possibilités pour que tous les enseignants puissent repenser leurs interventions. Dès lors, un travail d'opérationnalisation (dans le sens de « la mise en actes ") de ces valeurs sur le terrain semble primordial. Des réflexions autour des stratégies d'enseignement, mais aussi sur les postures professionnelles envisageables en fonction des registres de valeurs sont à mener. Un nouveau champ de réflexions s'ouvre pour la formation professionnelle et la connaissance scientifique. Ces perspectives semblent importantes pour tous les acteurs afin de viser les bénéfices objectivés par le bienêtre au travail. Elles permettent par ailleurs de développer la responsabilité des enseignants, en les rendant plus autonomes dans la conception et la régulation de leur propre stratégie d'intervention.

Avec la refonte des instituts de formation des enseignants en France, cette recherche participe à répondre aux nouveaux défis sociaux, nouveaux contextes et nouveaux enjeux dans l'enseignement en innovant dans la réflexion sur les pratiques d'intervention dans l'enseignement et plus particulièrement en EPS.

Travailler sur ses valeurs pour être en bien-être devrait permettre d'enclencher un cercle vertueux se répercutant positivement sur le comportement des élèves, leurs performances et leur bien-être (Renshaw et al., 2015 ; Rascle \& Bergugnat, 2016 ; OCDE, 2017). 


\section{eJRIEPS spécial 3 janvier 2020}

\subsection{Limites et perspectives}

Le paradigme dominant de cette étude aborde l'activité des enseignants d'EPS dans un cadre théorique en psychologique sociale.

D'autres approches d'orientations historico-culturelles (e.g., en ergonomie, en psychologie du travail et didactiques) peuvent être mobilisées pour traiter la question du bien-être au travail des enseignants. Ces études se basent sur des fondements théoriques et méthodologiques différents, mais paraissent tout aussi essentielles pour comprendre l'intervention en EPS et le bien-être des enseignants. D'autres variables agissent sans doute sur les liens entre le BE et le système de valeurs des enseignants telles que le parcours des enseignants, la valorisation perçue par l'enseignant, la situation de carrière ou l'organisation du travail. En effet, la spécificité du travail enseignant, du travail d'enseignant d'EPS, mais aussi du contexte culturel, politique, institutionnel situé dans lesquels l'activité de l'enseignant se réalise mériteraient d'être interrogés en parallèle pour avoir une vision plus englobante des relations entre les systèmes de valeurs et le bien-être. Canguilhem (2002) rappelle l'impact médiateur du contexte professionnel dans l'interdépendance entre l'activité professionnelle et la santé. Le rôle vertueux des systèmes de valeurs ne peut être dissocié des contextes (macro, méso et micro) dans lesquelles l'acteur intervient. Même si certaines variables ont été contrôlées, il semblera judicieux d'interroger ces autres approches, ainsi que les valeurs et les congruences à différents niveaux (Bronfenbrenner, 1979) dans de futurs travaux.

Par ailleurs, les interventions concrètes sont aussi faites d'empêchements, de renoncements qui mettent l'individu dans une position de tension entre ses buts et son activité (Leontiev, 1974). Ils ne relèvent pas toujours de son système de valeurs, mais de la confrontation entre ce qu'on lui demande de faire à ce que ça lui demande (Hubault, 2009). En effet, d'autres recherches (cf. en ergonomie, en didactique et en psychologie du travail) montrent la complexité des relations entre le bien-être et l'efficacité au travail. Ces travaux insistent sur les liens entre les multiples prescriptions (parfois contradictoires), les motifs d'engagement dans les situations de travail et les conditions effectives de travail (e.g., Falzon, 2013). Il sera intéressant de poursuivre la présente étude avec une prise en compte des conditions effectives de travail et des prescriptions qui pèsent sur les professeurs d'EPS.

Ces résultats tendent à être complétés pour étendre la réflexion à d'autres populations notamment dans d'autres disciplines d'enseignement. Par ailleurs, une autre ressource semble sous-exploitée, à savoir celle des congruences de valeurs (Skaalvik \& Skaalvik 


\section{eJRIEPS spécial 3 janvier 2020}

2011 ; Skaalvik \& Skaalvik 2015). Contrairement à l'approche par hiérarchie de valeurs, elle permet d'interroger si l'enseignant met en œuvre dans son enseignement des pratiques en cohérence avec son système général de valeurs.

\section{Conclusion}

L'objet de cette recherche était d'examiner le lien entre le système de valeurs des enseignants d'EPS et leur bien-être psychologique au travail. Des outils de mesure opérationnalisés ont été proposés dans le contexte professionnel de l'enseignement ainsi que celui de l'EPS en particulier. Ainsi, les systèmes de valeurs ont été étudiés dans différents contextes (SVG, SVens, SVeps).

Ce travail révèle trois pistes principales pour améliorer le bien-être psychologique au travail des professeurs d'EPS. La première est à envisager essentiellement dans le contexte général avec le domaine de l'ouverture au changement, effet préalablement démontré dans la littérature. Les deux suivantes sont ceux de l'affirmation de soi et du dépassement de soi en actes dans le contexte de l'EPS. Dès lors, l'identification des domaines de valeurs bénéfiques est une focale singulière et vertueuse pour repenser l'acte d'intervention et les stratégies déployées par les acteurs de l'EPS. En effet, en fonction de ses systèmes de valeurs (SVG, SVens, SVeps) l'enseignant est donc plus ou moins en bien-être psychologique au travail. Ainsi, l'approche par domaine de valeurs permet de repenser l'acte et les stratégies d'intervention des acteurs. Et même si les résultats restent limités aux enseignants d'EPS de l'Académie de Lille interrogés, ils ouvrent de nouvelles pistes de recherche pour la formation initiale et continue des enseignants d'EPS afin de répondre à ces nouveaux enjeux. Les valeurs sont dynamiques, elles varient en importance et sont inter-reliées entre elles (Schwartz et al., 2012). Le système de valeurs peut évoluer au gré des expériences et du contexte des individus (Schwartz et al., 2012). Face aux défis sociaux contemporains et à la recrudescence des difficultés professionnelles des acteurs dans l'enseignement (Nunez Moscoso, \& Murillo, 2017), cette recherche participe à développer une compréhension des facteurs qui contribuent au bien-être professionnel des enseignants pour repenser l'acte d'intervention notamment en EPS. Une des responsabilités de l'enseignant d'EPS pourrait être de prendre conscience de ses valeurs et de celles mises en œuvre dans son enseignement pour les adapter afin qu'elles contribuent à son bien-être et son efficacité enseignante. 


\section{eJRIEPS spécial 3 janvier 2020}

Remerciements : Nous tenons à remercier les enseignant.e.s d'EPS de l'Académie de Lille impliqué.e.s dans ce projet de recherche.

\section{Bibliographie}

Assael, H. (1984). Consumer behavior and marketing action (2nd ed). Boston : Kent Pub. Co.

Avallone, F., Farnese, M. L., Pepe, S., \& Vecchione, M. (2010). The Work Values Questionnaire (WVQ) : Revisiting Schwartz's Portrait Values Questionnaire (PVQ) for work contexts. BPA-Applied Psychology Bulletin (Bollettino di Psicologia Applicata), 261-262(57), 59-76.

Bardi, A., \& Schwartz, S. H. (2013). How does the value structure underlie value conflict. In J. Whitehead (Eds.), Values in youth sport and physical education, (pp. 137-151). London : Routledge.

Barrère, A. (2017). Au coeur des malaises enseignants. Paris : Armand Colin.

Biétry, F., \& Creusier, J. (2013). Proposition d'une échelle de mesure positive du bien-être au travail (EPBET). Revue de gestion des ressources humaines, 87(1), 23.

Bilsky, W., Janik, M., \& Schwartz, S. H. (2011). The structural organization of human valuesevidence from three rounds of the European Social Survey (ESS). Journal of CrossCultural Psychology, 42(5), 759-776.

Bronfenbrenner, U. (1979). The ecology of human development: experiments by nature and design. Cambridge, Mass: Harvard University Press.

Bruner, J. (2014). Pour une psychologie culturelle. In J.-F. Dortier (Eds.), Le cerveau et la pensée : le nouvel âge des sciences cognitives, (pp. 30-44). Paris : Éditions Sciences humaines.

Bryk, A. S. (2017). Accélérer la manière dont nous apprenons à améliorer. Education \& didactique, 11(2), 11-29.

Byrne, B. M. (2001). Structural Equation Modeling With AMOS, EQS, and LISREL: Comparative Approaches to Testing for the Factorial Validity of a Measuring Instrument. International Journal of Testing, 1(1), 55-86.

Carricano, M., Poujol, F., \& Bertrandias, L. (2010). Analyse de données avec SPSS. Paris : Pearson education.

Chang, M.-L. (2009). An appraisal perspective of teacher burnout: examining the emotional work of teachers. Educational Psychology Review, 21(3), 193-218. 


\section{eJRIEPS spécial 3 janvier 2020}

Cieciuch, J., Davidov, E., Schmidt, P., \& Algesheimer, R. (2016). Assessment of crosscultural comparability. In C. Wolf, D. Joye, T. Smith, \& Y. Fu (Eds.), The SAGE Handbook of Survey Methodology, (pp. 630-648). London: SAGE Publications.

Consiglio, C., Cenciotti, R., Borgogni, L., Alessandri, G., \& Schwartz, S. H. (2017). The WVal: A New Measure of Work Values. Journal of Career Assessment, 25(3), 405-422.

Coutarel, F., Mardon, C., Volkoff, S., Fiard, J., Récopé, M., Beaujouan, J., \& Rix, G. (2015). Enquête sur les conditions de travail auprès de professeurs d'éducation physique et sportive et perspectives d'action. Perspectives interdisciplinaires sur le travail et la santé, 17(1), 1-29.

Curby, T. W., Downer, J. T., \& Booren, L. M. (2014). Behavioral exchanges between teachers and children over the course of a typical preschool day: Testing bidirectional associations. Early Childhood Research Quarterly, 29(2), 193-204.

Dagenais-Desmarais, V., \& Privé, C. (2010). Comment améliorer le bien-être psychologique au travail ? Gestion, 35(3), 69.

Dagenais-Desmarais, V., \& Savoie, A. (2012). What is psychological well-being, really? A grassroots approach from the organizational sciences. Journal of Happiness Studies, 13(4), 659-684.

De Clercq, S., Fontaine, J. R. J., \& Anseel, F. (2008). In search of a Comprehensive value model for assessing supplementary person-organization fit. The Journal of Psychology, 142(3), 277-302.

Debarbieux, É. (2017). Ne tirez pas sur l'école! ...Réformez-la vraiment. Paris : Armand Colin.

Eccles, J. S., \& Roeser, R. W. (2011). Schools as developmental contexts during adolescence. Journal of Research on Adolescence, 21(1), 225-241.

Falzon, P. (2013). Ergonomie constructive. Paris: Presses Universitaire de France.

Ferrière, S., Bacro, F., Florin, A., \& Guimard, P. (2016). Le bien-être en contexte scolaire : Intérêt d'une approche par triangulation méthodologique. Les Cahiers Internationaux de Psychologie Sociale, 111(3), 341-365.

Fischer, R., \& Schwartz, S. (2011). Whence Differences in Value Priorities? Individual, Cultural, or Artifactual Sources. Journal of Cross-Cultural Psychology, 42(7), 1127-1144.

Florin, A., \& Guimard, P. (2017). Qualité de vie à l'école : comment l'école peut-elle proposer un cadre de vie favorable à la réussite et au bien-être des élèves ? Paris : CNESCO. 
eJRIEPS spécial 3 janvier 2020

Page consultée le 30 mars 2019 http://www.cnesco.fr/wpcontent/uploads/2017/10/170929 QDV FLorin Guimard.pdf.

Fraser, B. J. (1998). Classroom environment instruments : development, validity and applications. Learning Environments Research, 1(1), 7-34.

Galland, O., \& Lemel, Y. (2006). Tradition-modernité : un clivage persistant des sociétés européennes. Revue française de sociologie, 47(4), 687.

Gillies, R. M., \& Ashman, A. F. (2003). An historical review of the use of groups to promote socialization and learning. In R. M. Gillies \& A. F. Ashman (Eds.), Co-operative learning: The social and intellectual outcomes of learning in groups, (pp. 1-18). New York, NY: Routledge.

Guibert, P. (2017). L'accueil des nouveaux enseignants dans les collèges et les lycées français. Revue internationale d'éducation de Sèvres, (74), 105-114.

Hitlin, S., \& Piliavin, J. A. (2004). Values : reviving a dormant concept. Annual Review of Sociology, 30(1), 359-393.

Hooper, D., Coughlan, J., \& Mullen, M. R. (2008). Structural equation modelling : guidelines for determining model fit. Electronic Journal of Business Research Methods, 6(1), 5360.

Hu, L., \& Bentler, P. M. (1999). Cutoff criteria for fit indexes in covariance structure analysis: Conventional criteria versus new alternatives. Structural Equation Modeling : A Multidisciplinary Journal, 6(1), 1-55.

Hubault, F. (2009). L'approche ergonomique des questions santé / travail. Mouvements, 58(2), 97.

Jégo, S., \& Guillo, C. (2016). Les enseignants face aux risques psychosociaux : Comparaison des enseignants avec certains cadres du privé et de la fonction publique en 2013. Éducation et formations, (92), 77-113.

Jewett, A. E., Bain, L. L., \& Ennis, C. D. (1995). The curriculum process in physical education (2nd edition). Madison, WI: Brown \& Benchmark.

Karsenti, T., Collin, S., \& Dumouchel, G. (2013). Le décrochage enseignant: état des connaissances. International Review of Education, 59(5), 549-568.

Kashdan, T. B., Biswas-Diener, R., \& King, L. A. (2008). Reconsidering happiness: the costs of distinguishing between hedonics and eudaimonia. The Journal of Positive Psychology, 3(4), 219-233.

Kursa, M. B., \& Rudnicki, W. R. (2010). Feature selection with the boruta package. Journal of Statistical Software, 36(11). 


\section{eJRIEPS spécial 3 janvier 2020}

Le Bot, G., Pasco, D., \& Desbiens, J.-F. (2011). Des orientations de valeur dans les programmes à leur mise en oeuvre en classe. Spiral-E. Revue de recherches en éducation, supplément électronique, 47(1), 51-74.

Le Bot, G., Desbiens, J.-F., \& Pasco, D. (2016). Identifier les orientations de valeur des enseignants à partir de l'analyse de leur pratique d'enseignement: conception et validation d'une grille d'observation en éducation physique et sportive. eJRIEPS, (39), 111-135.

Leontiev, A. N. (1974). The Problem of Activity in Psychology. Soviet Psychology, 13(2), 4-33.

Loup, P. (2016). Influence des technologies nomades sur le bien-être au travail: une lecture par la théorie de la conservation des ressources. Thèse de doctorat de sciences de gestion. Université de Montpellier, Montpellier, France.

Lyubomirsky, S., King, L., \& Diener, E. (2005). The benefits of frequent positive affect : does happiness lead to success? Psychological Bulletin, 131(6), 803-855.

Nunez Moscoso, J., \& Murillo, A. (2017). La difficulté dans le travail enseignant : un thème de recherche aux objets pluriels. Penser l'éducation, (40), 59-87.

OCDE. (2014). Guide TALIS 2013 à l'intention des enseignants : Enquête internationale sur l'enseignement et l'apprentissage. Paris : OECD Publishing.

OCDE. (2017). PISA 2015 results: students' well-being (Vol. III). Paris : OECD Publishing.

Ochoa, P., \& Blanch, J. (2018). Psychosocial well-being at work: reasons to invest in healthy employees and workplaces. In P. Ochoa \& M. T. Lepeley, P. Essens (Eds.), Wellbeing for sustainability in the global workplace, (pp. 10-23). London : Routledge.

Rascle, N., \& Bergugnat, L. (2016). Qualité de vie des enseignants en relation avec celle des élèves : revue de question, recommandations. Paris : CNESCO. Page consultée $\begin{array}{llll}\text { le } & 30 & \text { mars } & 2019 \\ \text { http://www.cnesco.fr/wp- }\end{array}$ content/uploads/2017/10/170929 enseignants.pdf.

Rayou, P. (2017). S'améliorer pour améliorer l'école des réseaux et des savoirs. Sur l'ouvrage d'Anthony S. Bryk, Accelerating How We Learn to Improve. Education \& didactique, 11(2), 39-43.

Reboul, O. (1992). Les valeurs de l'éducation. Paris : Presses Universitaires de France.

Reboul, O. (2016). La philosophie de l'éducation. (11ème edition). Paris : Presses Universitaires de France. 


\section{eJRIEPS spécial 3 janvier 2020}

Renshaw, T. L., Long, A. C. J., \& Cook, C. R. (2015). Assessing adolescents' positive psychological functioning at school : development and validation of the student subjective well-being questionnaire. School Psychology Quarterly, 30(4), 534-552.

Retelsdorf, J., Butler, R., Streblow, L., \& Schiefele, U. (2010). Teachers' goal orientations for teaching : associations with instructional practices, interest in teaching, and burnout. Learning and Instruction, 20(1), 30-46.

Rezsohazy, R. (2006). Sociologie des valeurs. Paris : Armand Colin.

Ros, M., Schwartz, S. H., \& Surkiss, S. (1999). Basic individual values, work values, and the meaning of work. Applied Psychology, 48(1), 49-71.

Sagiv, L., Roccas, S., \& Hazan, O. (2004). Value pathways to well-being : healthy values, valued goal attainment, and environmental congruence. In P. A. Linley \& S. Joseph (Eds.), Positive Psychology in Practice, (pp. 68-85). Hoboken, N.J. : John Wiley \& Sons, Inc.

Sagiv, L., \& Schwartz, S. H. (2000). Value priorities and subjective well-being : direct relations and congruity effects. European Journal of Social Psychology, 30(2), 177-198.

Sahlberg, P. (2010). Rethinking accountability in a knowledge society. Journal of Educational Change, 11(1), 45-61.

Schleicher, A. (2018). Valuing our teachers and raising their status : how communities can help. International summit on teaching profession. Paris : OECD Publishing.

Schoëndorff, B., Grand, J., \& Bolduc, M. (2011). La thérapie d'acceptation et d'engagement : guide clinique. Bruxelles : De Boeck.

Schwartz, S. H. (1992). Universals in the Content and Structure of Values: Theoretical Advances and Empirical Tests in 20 Countries. In M. P. Zanna, Advances in Experimental Social Psychology, (pp.1-65). San Diego : Academic Press.

Schwartz, S. H. (1997). Motivation and culture. In D. Munro, J. F. Schumaker, \& S. C. Carr (Eds.), Values and culture, (pp. 69-84). New York : Routledge.

Schwartz, S. H. (2003). A proposal for measuring value orientations across nations. Questionnaire Package of the European Social Survey, 259-290.

Schwartz, S. H. (2006). Les valeurs de base de la personne: théorie, mesures et applications. Revue française de sociologie, 47(4), 929.

Schwartz, S. H., \& Bardi, A. (2001). Value hierarchies across cultures : taking a similarities perspective. Journal of Cross-Cultural Psychology, 32(3), 268-290.

Schwartz, S. H., \& Bilsky, W. (1987). Toward a universal psychological structure of human values. Journal of Personality and Social Psychology, 53(3), 550-562. 


\section{eJRIEPS spécial 3 janvier 2020}

Schwartz, S. H., \& Bilsky, W. (1990). Toward a theory of the universal content and structure of values : Extensions and cross-cultural replications. Journal of Personality and Social Psychology, 58(5), 878-891.

Schwartz, S. H., Melech, G., Lehmann, A., Burgess, S., Harris, M., \& Owens, V. (2001). Extending the cross-cultural validity of the theory of basic human values with a different method of measurement. Journal of Cross-Cultural Psychology, 32(5), 519-542.

Schwartz, S. H., Cieciuch, J., Vecchione, M., Davidov, E., Fischer, R., Beierlein, C., Ramos, A., Verkasalo, M., Lönnqvist, J. E., Demirutku, K., Dirilen-Gumus, O. \& Konty, M. (2012). Refining the theory of basic individual values. Journal of Personality and Social Psychology, 103(4), 663-688.

Skaalvik, E. M., \& Skaalvik, S. (2011). Teacher job satisfaction and motivation to leave the teaching profession : Relations with school context, feeling of belonging, and emotional exhaustion. Teaching and Teacher Education, 27(6), 1029-1038.

Skaalvik, E. M., \& Skaalvik, S. (2015). Job satisfaction, stress and coping strategies in the teaching profession-what do teachers say ? International Education Studies, 8(3).

Sortheix, F. M., \& Schwartz, S. H. (2017). Values that underlie and undermine well-being: variability across countries: values that underlie and undermine well-being. European Journal of Personality, 31(2), 187-201.

Tolmie, A. K., Topping, K. J., Christie, D., Donaldson, C., Howe, C., Jessiman, E., Livingston, K., Thurston, A. (2010). Social effects of collaborative learning in primary schools. Learning and Instruction, 20(3), 177-191.

Wilson, K. G., \& DuFrene, T. (2008). Mindfulness for two: an acceptance and commitment therapy approach to mindfulness in psychotherapy. Oakland : New Harbinger Publications. 